AsCLEPIO. Revista de Historia de la Medicina y de la Ciencia

66 (2), julio-diciembre 2014, p056

ISSN-L:0210-4466

http://dx.doi.org/10.3989/asclepio.2014.20

DOSSIER: BIOPODER Y DETERMINISMOS EN SUDAMÉRICA DURANTE EL SIGLO XX / DOSSIER: BIOPOWER AND DETERMINISM IN SOUTH AMERICA DURING THE TWENTIETH CENTURY

\title{
UNA DIALÓGICA DESESTABILIZADORA DEL ORDEN SOCIAL Y SEXUAL: EL MÉDICO ARGENTINO JUAN LAZARTE EN LA REVISTA ANARQUISTA ESTUDIOS (1932-1936)
}

\author{
Isabel Jiménez-Lucena \\ Universidad de Málaga \\ isajimenez@uma.es \\ Jorge Molero-Mesa \\ Universidad Autónoma de Barcelona \\ Jorge.Molero@uab.cat
}

Recibido: 9 septiembre 2013; Aceptado: 14 abril 2014.

Cómo citar este artículo/Citation: Jiménez-Lucena, Isabel y Jorge Molero-Mesa, (2014), “Una dialógica desestabilizadora del orden social y sexual: el médico argentino Juan Lazarte en la revista anarquista Estudios (1932-1936)”, Asclepio, 66 (2): p056. doi: http://dx.doi. org/10.3989/asclepio.2014.20

RESUMEN: En este trabajo analizamos los artículos del médico argentino Juan Lazarte publicados en la revista Estudios en los años 30 del siglo XX. Este hecho se produjo en el marco de una política editorial que pretendía superar la exclusión a que eran sometidos los discursos de oposición al orden social establecido. La práctica discursiva analizada se desarrolló sobre una dialógica en torno a las propuestas neomaltusianas y eugenésicas y a las causas históricas de unas relaciones sociales generizadas que estructuraban una doble moral sexual. Lazarte usó diversas formas de intertextualidad y señaló la compatibilidad de planteamientos que se mostraban opuestos en apariencia, sin obviar las diferencias, evitando los debates en términos dicotómicos. Esta praxis hizo posible un uso táctico de la retórica eugenésica en la propuesta neomaltusiana con la que se pretendía que la especie humana alcanzase un estado de buen vivir. Al no concebir la historia desde una ideología del progreso, y empeñarse en considerar los distintos aspectos de los problemas (biológicos, económicos y culturales), el discurso de Lazarte en Estudios contribuía a conformar un mundo que desafiaba los supuestos de un biopoder determinista, constituyéndose en un poder desestabilizador del orden sexual y social.

PALABRAS CLAVE: Anarquismo Libertario; Neomaltusianismo; Eugenesia; Liberación Sexual; Gestión del Conocimiento.

\section{A DESTABILIZING DIALOGICS OF SOCIAL AND SEXUAL ORDER: THE ARGENTINIAN PHYSICIAN JUAN LAZARTE IN THE ANARCHIST MAGAZINE ESTUDIOS (1932-1936)}

ABSTRACT: In this paper we analyze the articles published by Argentinean physician Juan Larzarte in the magazine Estudios in the 1930s. This took place within a publishing policy aimed at overcoming the exclusion of discourses opposing the established social order. The discourse practices we explore were dialogic while focusing on neo-Malthusian and eugenic propositions and the historical causes of a gendered social relations structuring a double sexual morals. Lazarte used several forms of intertextuality and suggested the compatibility of seemingly opposed approaches without getting round the differences and avoiding debates in dichotomic terms. These practices allowed the strategic use of eugenic rhetoric within the neo-Malthusian proposition aimed at the human species to reach a state of good living. As he did not consider history from an ideology of progress, and insisted on taking into account the different facets of any issue (biological, economic and cultural), Lazarte's discourse in Estudios contributed to configure a world defying the assumptions of a deterministic biopower, thus becoming a destabilizing power regarding the sexual and social order.

KEY WORDS: Libertarian Anarchism; Neo-Malthusianism; Eugenics; Sexual Liberation; Knowledge Management.

Copyright: () 2014 CSIC. Este es un artículo de acceso abierto distribuido bajo los términos de la licencia Creative Commons Attribution-Non Commercial (by-nc) Spain 3.0. 


\section{INTRODUCCIÓN}

Es bien conocido que durante los años treinta del siglo XX la eugenesia y el neomaltusianismo ocuparon un lugar relevante en el orden discursivo de las sociedades occidentales ${ }^{1}$. Sin embargo, no todos los discursos interpretaron de la misma manera los saberes y las prácticas eugenésicas y neomaltusianas, sino que existieron discursos dominantes y discursos de oposición que incorporaron y transformaron sus significados ${ }^{2}$. Aunque, como ha puesto de manifiesto el análisis crítico del discurso, el poder establecido pretende el control de la producción y circulación de los discursos a través de distintos procedimientos entre los que se encuentra el de la exclusión de discursos de oposición, alternativos y disidentes ${ }^{3}$, en las primeras décadas del siglo XX fueron posibles en el ámbito español y argentino iniciativas editoriales anarcolibertarias que formaron parte de un programa cultural y educativo integrante de una estrategia revolucionaria de transformación social ${ }^{4}$. Producir conocimiento desde la praxis y para la praxis fue una empresa prioritaria para el movimiento anarquista ${ }^{5}$. Con ello se pretendía propagar las ideas libertarias, pero también ofrecer instrumentos para el análisis crítico de la sociedad. En este empeño se pusieron en marcha editoriales y publicaciones periódicas que tanto en España como en Argentina se proponían dar a conocer las bases de una sociedad alternativa a la capitalista, sin divisiones sociales y autogestionada ${ }^{6}$. Entre las herramientas que posibilitarían la construcción de esa sociedad se dispuso de revistas como Estudios. Revista Ecléctica (Barcelona, 1928-1937) que representó el espacio idóneo de discusión para el anarquismo que tomaba el eclecticismo como base y fundamento del conocimiento, social e ideológicamente interesado ${ }^{7}$. Estudios se publicó en el periodo histórico considerado "la época dorada de la prensa cultural anarquista", y fue uno de los proyectos editoriales más exitosos del movimiento libertario español (Díez, 2007, pp. 102-110). Xavier Díez la considera "la revista cultural más trascendente y prestigiosa del anarquismo español" además de la más vendida, y apunta que una de las razones de este éxito fue el carácter abierto y ecléctico de la revista que consiguió el apoyo de las diversas orientaciones ácratas (Díez, 2007, pp. 132-138). Estudios perseguía objetivos compartidos por la prensa anarcolibertaria: informar, divulgar y debatir para construir un sistema alternativo de valores coherentes con la visión del mundo y la forma de vida libertarias que pondrían las bases para una economía, una sociedad y una cultura alternativas (Navarro, 1997; Díez, 2007). Esta publicación contó con una gran proyección hacia los países latinoamericanos y de forma especial hacia Argentina, país en el que se pudieron distribuir más de veinte mil ejemplares ${ }^{8}$. En ella colaboraron autores como el médico argentino Juan Lazarte (Rosario, Argentina 18911963). Este autor estuvo vinculado desde muy joven a ambientes libertarios ${ }^{9}$ siendo su maestro de estudios primarios Julio Barcos ${ }^{10}$; sus inquietudes intelectuales le llevaron a obtener una formación amplia como naturalista, genetista y médico ${ }^{11}$. Su activismo militante revolucionario le condujo a comprometerse con las luchas estudiantiles y obreras y a participar en diferentes publicaciones anarquistas (Ledesma, 2010b y Graciano, 2012). Desarrollando algunas de las líneas propuestas por Barcos ${ }^{12}$, mostró un especial interés por el conocimiento científico de problemas sociales como la situación de las mujeres y las relaciones de género así como el control de la natalidad. En este sentido, pensamos que el análisis en detalle de las publicaciones de Lazarte en Estudios puede acercarnos más a la comprensión del papel que la revista quería jugar en la apropiación de los distintos significados científicos no sólo transmitiendo el conocimiento sino, también, (re)significándolo para transformarlo en una herramienta de cambio revolucionario. En este trabajo analizaremos los cinco artículos de Juan Lazarte publicados en Estudios entre los años 1932 y 1936 : "Desprestigio del adulterio" (Lazarte, 1932), "Significación cultural y ética de la limitación de los nacimientos" (Lazarte, 1933a-e, 1934a-c), "La atrofia de la sensibilidad en las mujeres" (Lazarte, 1935a), "Los tiempos agenésicos" (Lazarte, 1935b) y "Milagros y Medicina" (Lazarte, 1936).

Una primera aproximación a estos textos nos muestra cómo la significación conflictiva del neomaltusianismo y la eugenesia se plasmó en el discurso de Lazarte que sólo utilizó el término "eugenesia" y sus derivados en cuatro ocasiones y "neomaltusianismo" en seis aunque en este caso le dedicó un epígrafe completo a aclarar las diferencias entre las propuestas de Malthus y el neomaltusianismo. Ahora bien, cabe preguntarse si, aun así, dada la temática de los trabajos publicados, estuvieron presentes de forma significativa la eugenesia y el neomaltusianismo en el discurso de Lazarte en Estudios. La respuesta a esta cuestión es que ambas propuestas ocuparon un lugar destacado en los contenidos abordados y que, como veremos en las páginas que siguen, fueron utilizadas con unos fines emancipadores en el marco de una praxis discursiva esencialmente dialógica que formaba parte de una práctica de resistencia y transformadora frente a un biopoder determinista.

\section{LA AUTORIDAD DE JUAN LAZARTE EN LA REVISTA ESTUDIOS}

Podemos decir que existía una verdadera sintonía de la revista Estudios como espacio de discusión y Lazarte como profesor que, siguiendo la tradición fomentadora del análisis y debate de las controversias en el anarquismo argentino ${ }^{13}$, "trataba de promover búsqueda y más que exponer doctrina quería que sus alumnos conquistaran pensando sus propias convic- 
ciones [...] no se cansaba nunca de recomendar lecturas, de encargar monografías, de suscitar discusiones, de promover, aunque fuera dentro de los estrechos límites de un aula, la investigación" (Cappelletti, 1964, pp. 37-38 $)^{14}$.

Como hemos señalado antes, la política editorial libertaria pretendía superar la exclusión a que eran sometidos los discursos de oposición al orden social. En este sentido, a la vez que se reconocía la importancia del discurso en la transformación de la sociedad como un elemento de la praxis social ${ }^{15}$, había una consciencia de que la trascendencia social de los discursos dependía del grado de reproducción de los mismos, de las citas que recibieran, de su distribución social.

En esta línea, la obra de Juan Lazarte fue difundida por distintos colaboradores de Estudios. Así, Maria Lacerda de Moura (1897-1945) utilizó las estadísticas de Lazarte en su artículo neomaltusiano "Procreación y miseria":

Lamento, asimismo, no poder transcribir otros datos demostrativos de que el exceso de nacimientos acarrea inevitablemente la crecida mortalidad infantil. Veamos, no obstante, las conclusiones de una estadística que me proporcionó el ilustre médico argentino doctor Juan Lazarte, uno de los más dignos y nobles «camaradas» nuestros.

¡Por cada 7.389 niños que nacen, mueren 3.451, o sea, casi la mitad! [...]Hamburger, de Alemania, cita una estadística personal, semejante a la que me proporcionó el doctor Lazarte, aunque algo atenuada. "La mortalidad infantil - dice- en las familias que sólo tienen un hijo es de un $23 \%$, mientras que en los hogares de ocho hijos se eleva a $51 \%$, alcanzando el $69 \%$ en aquellos casos en que la prole va de 10 a 15" (Lacerda, 1932)

La autoridad de Lazarte en temas relacionados con los cambios en la sexualidad ${ }^{16}$ fue reconocida por $\mathrm{Hi}$ ginio Noja Ruiz (1896-1972), colaborador habitual de la revista, al reseñar el libro La revolución sexual de nuestro tiempo, editado en 1932 por Ediciones Nervio en Buenos Aires:

No se puede tratar en un volumen de las reducidas dimensiones de éste con más amplitud y suficiencia tema tan interesante y complejo. Bien es verdad que el doctor Lazarte no es, como escritor y como sociólogo, cualquier cosa. Este folleto le acreditaría si no estuviera ya suficientemente acreditado. Todo lo que se refiere a la revolución que en el terreno del sexualismo se viene operando en el mundo en nuestra época, está tratado en este Cuaderno con una pericia y una precisión, rara vez mejor lograda. Ni un solo aspecto de la cuestión es echado en olvido. $Y$ en todas las páginas, al lado del rigorismo científico, hallamos la limpieza de estilo, y la solidez de juicios del escritor y del sabio. Reciban, autor y editores, el homenaje de nuestras simpatías y nuestra cordial enhorabuena (H.N.R., 1932).
También se reseñaron en la sección "Bibliografía" los trabajos de Lazarte sobre la socialización de la medicina y la reforma universitaria, considerándolos "una aportación seria a la causa de la cultura" (Anónimo, 1934) y reconociendo en el doctor Juan Lazarte "un trabajador infatigable" (García, 1936).

Félix Martí Ibáñez (1911-1972) asignaba a Lazarte el concepto de "proletarización del médico" en la crítica al funcionamiento de "las empresas particularistas que hacían de la Sanidad un instrumento al servicio de sus propios intereses" (Martí, 1937).

Por tanto, se presentaba a Lazarte como una autoridad en la elaboración de conocimientos que llevarían a la construcción de alternativas necesarias para el cambio del sistema social vigente, confiriéndosele un estatus de autoridad en la práctica discursiva anarcolibertaria que representaba la revista Estudios. De esta forma, la obra de Juan Lazarte publicada en Estudios jugó un relevante papel en la acción legitimadora y reivindicativa de la limitación de los nacimientos y de la liberación de las mujeres.

\section{SIGNIFICACIÓN LIBERTARIA DE LA LIMITACIÓN DE NACIMIENTOS}

La apuesta neomaltusiana de la revista Estudios fue clara. Es cierto que no todos los integrantes del movimiento libertario apoyaban esta opción ${ }^{17}$, hecho que se recogía en los textos de relevantes colaboradores de Estudios que se apoyaron en datos suministrados por Lazarte, como fue el caso de Maria Lacerda de Moura:

No hay despoblación. Lo que sí existe en todo el mundo es sobrepoblación. Ante las lamentables consecuencias de semejante estado de cosas, no podemos menos que estremecernos pensando en la actitud hostil de muchos revolucionarios, Kropotkin le decía a Paul Robin: «Estás estorbando el advenimiento de la Revolución.» James Guillaume escribíale: «Eres un obstáculo para la emancipación del obrero». Y Elíseo Reclús negábase a publicar en su periódico los artículos neomaltusianos, so pretexto de que este asunto era de índole particular, y que, desde el punto de vista general, la limitación de nacimientos era tan sólo una «gran mixtificación».

Ante toda esta coaligación de juicios y actividades adversas, no podemos por menos que sentir profunda admiración hacia ese gran hombre que se llamó Paul Robin, cuya perseverancia igualaba a la magnitud de sus sentimientos fraternales. Si todos los anarquistas se hubiesen tomado el trabajo de estudiar este asunto con el detenimiento y la imparcialidad que lo ha hecho Sebastián Faure, como él habrían cambiado de opinión (Lacerda, 1932).

En esta disputa Lazarte fue un neomaltusiano convencido, que dedicó buena parte de su obra escrita a propagar ideas y prácticas sobre el control voluntario 
de la natalidad. En la publicación de las ocho partes que Estudios hizo del trabajo de Lazarte "Significación cultural y ética de la limitación de los nacimientos" (Lazarte, 1933a-e, 1934a-c) ${ }^{18}$ se abordaba la dimensión histórica del problema, considerando el control de la natalidad como un movimiento de liberación principalmente femenino, pero también masculino. Se hizo mención al medio siglo de lucha que sobre problemas sexuales se había desarrollado y cómo esa lucha había llevado hasta una nueva consciencia sexual que estaba construyendo un "nuevo mundo sexual" en países como Rusia y Norteamérica e incluso en Alemania donde el pueblo se enfrentaba al Estado fascista, constituyendo la disminución de los nacimientos todo un desafío al poder (Lazarte, 1933a). De esta forma, Lazarte participaba de la consideración del movimiento neomaltusiano como un instrumento político. Lazarte sostenía que en los países latinos estaban más atrasados en este asunto y aunque en España las "condiciones prerrevolucionarias" llevarían en pocos años a que se conocieran y discutieran los problemas sexuales, en Hispano América se mantenía una situación colonial debido a la existencia de unos "sectores burgueses, intelectuales y religiosos de cruda e infranqueable oscuridad", aunque "minorías de hombres de alta consciencia" trabajaban para cambiar esa situación (Lazarte, 1933a).

Junto a estos aspectos históricos se fueron exponiendo las razones científicas y morales que debían plantearse sobre este asunto. Lazarte desarrolló tres líneas argumentales, relacionadas con la medicalización del trabajo reproductivo, cuestiones de herencia y eugenesia y la liberación de las mujeres. Trataremos en este epígrafe las dos primeras y abordaremos la última en el siguiente apartado dedicado a la revolución sexual.

Ya abordamos en trabajos anteriores el papel que la medicalización de los aspectos reproductivos había jugado en la estrategia del activismo neomaltusiano (Jiménez y Molero, 2009; y Jiménez y Molero, 2011). En el caso de Lazarte se optó por desarrollar la idea del parto como catástrofe fisiológica, aunque el médico argentino, consciente de "la importancia que las sociedades dan a la maternidad" (Lazarte, 1933b), puso especial cuidado en evitar un enfrentamiento frontal con posiciones maternalistas mixtificadoras:

Un parto es una verdadera catástrofe fisiológica. No queremos hacer una antipropaganda de la maternidad, ni condenarla así porque sí en conjunto groseramente. El espectáculo más emocionante de la vida humana es el momento en que una mujer da a luz a un niño... Aquí caben todos los elogios y aplausos junto al más grande sentimiento de respeto y de religiosidad verdadera. Por de pronto, la maternidad es uno de los instintos básicos, no sólo humanos, sino animales y vegetales, en esto, en lo tocante a la reproducción (Lazarte, 1933a).
También sostenía que "jamás en la historia" se había defendido y protegido la maternidad como en 1932 y como se defendería y perfeccionaría en el futuro, debido a "los proyectos, leyes y pensamientos ya divulgados, que van tomando forma concreta en las novísimas legislaciones. De donde la maternidad no está en peligro, la reproducción ha tomado seguridades desconocidas en el convivir societario antiguo" (Lazarte, 1933b), tranquilizando de este modo a los pronatalistas.

Sin embargo, a pesar de estas precauciones, Lazarte consideraba que el parto suponía una "catástrofe" anatomofisiológica, psicológica e histórica para las mujeres (Lazarte, 1933a). Lazarte utilizó el concepto de "tragedia biológica de la mujer" aunque, consideraba que dicha tragedia, "si no vencida, pudiera ser atenuada por creaciones de la naturaleza societaria del hombre y aportes de la inteligencia individual" y planteaba que había diversas y encontradas opiniones sobre este asunto. Señalaba que existían importantes aspectos históricos que habían llevado a "la mujer" a una situación de doble esclavitud: esclavitud económica y esclavitud sexual. Para Lazarte la situación económica estaba muy vinculada a la mala situación higiénica de las mujeres obreras y por tanto a resultados de alto índice de mortalidad maternal, abortos y partos prematuros:

el parto no sólo es un peligro natural sino que está determinado en su peligrosidad por las causas económicas de la mujer, tanto como por las causas de la constitución fisiológica, y si a esta constitución orgánica unimos otras causas también económicas que antes sobre ella influyen, llegamos a la conclusión que para la mujer trabajadora un embarazo o un parto es asunto de suma importancia y también un problema unido a su liberación económica y social (Lazarte, 1933a).

Aportaba datos en los que en condiciones de trabajo doméstico se producían abortos y partos prematuros en proporciones sólo superiores a las del trabajo en fábricas con metales (plomo) (Lazarte, 1933a). Ésta era una de las maneras en que Lazarte vinculaba los riesgos reproductivos con la estructura de clases sociales. Aseguraba que existía una "íntima relación" entre el índice de mortalidad y el salario de los padres y para probarlo citaba los estudios del Children's Bureau de los Estados Unidos de Norteamérica publicados en el libro de Ernesto Nelson (1873-1959) La salud y el niño en Nueva York en 1929. Afirmaba que había "muchos oficios obreros que son causas directas -ya comprobadas- de mortalidad infantil" (Lazarte, 1933e). Así, "el imperativo de la anticoncepción" se fundamentaba en la alta mortalidad infantil y también en la mala salud de las mujeres como consecuencia de partos muy frecuentes. Para argumentar esto, Lazarte utilizaba diferentes casos descritos por Marie Carmichael Stopes (1880-1958) y Jane Hawthorne (m. 1945), entre ellos el siguiente relato de "una madre": 
Quisiera saber cómo evitar el tener más hijos, pues me parece que ya he cumplido mis deberes con la patria, por haberle dado trece hijos, nueve varones y cuatro hembras. Me viven seis niños y una niña que en mayo cumplirá tres años. Hace tres semanas se me murió una hija de tos ferina, y digo todo esto porque no puedo cuidar a mis pequeñuelos como quisiera... Como ni mi marido ni yo somos viejos, pues él cumplirá cuarenta años en julio próximo y yo cumplí treinta y nueve el 19 de febrero pasado, temo tener más hijos. Nos casamos hace veinte años y puede usted ver por los hijos que he tenido que no me ha quedado mucho tiempo para divertirme. Ahora estoy muy enferma de varices y apenas me valgo (Lazarte, 1934a).

Lazarte concluía: "casos como éstos no son excepciones. Los hay por cientos en todas partes. Los médicos prácticos han visto desfilar muchísimos y los parteros y ginecólogos no tienen más que consultar sus archivos para ofrecer al lector numerosísimos ejemplos"; por tanto, la anticoncepción era considerada "una necesidad pública" (Lazarte, 1934a).

Lazarte trataba de explicar por qué se había llegado a esa situación sosteniendo que era la ignorancia la que llevaba a la maternidad continua que además de suponer una nueva esclavitud era antihigiénica ya que los embarazos múltiples y seriados provocaban el agotamiento y la enfermedad en las mujeres (Lazarte, 1933c). Por otra parte, intentaba profundizar en las causas histórico-culturales que mantenían una actitud antineomaltusiana, señalando que una fuente de autoridad cultural como La Biblia había impuesto no sólo el "crecer y multiplicaos" sino el parir con dolor como fuente de dicha (Lazarte, 1933a), y ahondando en esto el cristianismo había realizado "un supremo esfuerzo de destrucción de la recreación" (Lazarte, 1933b). Sin embargo, este contexto cultural no había sido ni era el único posible como se encargó Lazarte de mostrar ${ }^{19}$. El aspecto recreativo del amor había sido considerado por las culturas occidentales precristianas y había sido "redescubierto" por la ciencia moderna, de manera que se podía disociar reproducción y recreación en lo que se refería a la actividad sexual humana (Lazarte, 1933b) ${ }^{20}$.

Esta realidad había llevado a la existencia, en todos los tiempos y lugares, de "antecedentes de limitación de nacimientos", antecedentes que servían a Lazarte para argumentar la necesidad de introducir el control y la voluntad en los aspectos reproductivos, pues "solamente a un troglodita se le ocurre pensar que se reproducen mejor los salvajes de Hotentosia (naturalmente) que las supercivilizadas jóvenes alemanas o rusas de las grandes ciudades, que ya están en posesión de los conocimientos anticoncepcionales" (Lazarte, 1933b). De este modo, los anticonceptivos se constituirían en "un factor activo y cooperador de la civilización" (Lazarte, 1933c). De ahí que hiciera una intensa crítica a las políticas natalistas y al concepto de buena madre que éstas difundían, asegurando que, por el contrario "No es la mejor madre la que tiene más hijos; seguramente está entre las peores" (Lazarte, 1933c). Ya había anunciado con anterioridad que la humanidad había conseguido transformar la sexualidad al considerar el aspecto recreativo del amor y dando otra perspectiva a su aspecto reproductivo la cual encerraba "una nueva maternidad" (Lazarte, 1933b). Además, formando parte de ese discurso opositor y resistente a la visión tradicional de la reproducción, Lazarte apuntaba que los asuntos reproductivos, y, por tanto el parto, debía ser considerado un bien social y como tal un trabajo productivo merecedor de retribución, de ahí que hiciese la siguiente referencia: "Bernard Shaw da tanta importancia al parto que cree se debe pagar a cada madre 20.000 dólares por hijo, dado los riesgos que en cada uno corre su vida y su cuerpo" (Lazarte, 1933a).

Junto a estos problemas de orden somático, Juan Lazarte sostenía que era necesario luchar contra los trastornos psíquicos causados por la idea del pecado y el miedo al embarazo. Éstos habían provocado temores y frenos que conducían a la disminución de la sensibilidad y neutralización de la fantasía. Estas circunstancias había desencadenado trastornos y degeneración del organismo, a las que Freud Ilamaba histeria y otros autores neurosis, que conducían a la insensibilidad e insatisfacción sexual causantes de, entre otros males, los celos (Lazarte, 1933b); la frigidez femenina era otra consecuencia de esta situación (Lazarte, 1935a) como veremos más adelante. Dada esta realidad, Lazarte proponía como solución una adecuada educación sexual y la enseñanza de los contraconceptivos que supondrían una ventaja para el organismo de las mujeres, las buenas relaciones de las uniones y el bienestar psicosocial de la colectividad. Estos grandes beneficios individuales y colectivos permitía a Lazarte propugnar un uso generalizado de contraconceptivos para solteras, recién casadas, casadas, madres, en definitiva para todas las mujeres en edad reproductiva (Lazarte, 1933b). Así, un objetivo de Lazarte era ayudar a las mujeres en la lucha por la anticoncepción dado que

Existen muchísimas mujeres, muy inteligentes, que en nuestra civilización colonial y precultural luchan como leonas para limitar la maternidad, topándose con la estupidez inmensa de los "maridos», el consenso que los anticonceptivos son condenados por la Iglesia, la dificultad de una información seria y científica, la pobreza y demás. Estos pobres seres son dignos de liberación. No quieren más hijos. Muchas, nos dicen: «Doctor, estoy harta de hijos y no sé cómo hacer» (Lazarte, 1933b).

Por ello, se preocupó Lazarte de difundir métodos anticonceptivos publicando información sobre el que se popularizó como método Ogino (Lazarte, 1935b). 
Consideraba que éste sería un importante avance en la anticoncepción, dado que las investigaciones en curso acabarían comprobando la existencia de un tiempo agenésico para toda mujer, lo que reportaría "el más alto beneficio humano" (Lazarte, 1935b). Pero, advertía de las dificultades que en aquel momento apreciaba en ese método anticonceptivo dado que "el período de fecundabilidad de la mujer es bastante complicado a determinar", por la variabilidad, y debido a ello "hay que andar con sumas precauciones" (Lazarte, 1935b). En su opinión "en todos los fenómenos analizados no existe una exactitud matemática como para fiarnos en absoluto [...] Aún faltan muchos puntos que aclarar en este método" (Lazarte, 1935b). Para entender el interés por un método que provocaba tantas dudas, no debemos olvidar la ventaja que tenía un coste económico nulo para las clases sociales más desfavorecidas.

Estrechamente relacionado con la medicalización de los asuntos reproductivos, la herencia y vinculada (o no) a ella la eugenesia ${ }^{21}$ estuvieron muy presentes en el ámbito discursivo de las primeras décadas del siglo XX. Como apuntábamos más arriba, la obra de Lazarte no fue una excepción. Lazarte en su defensa de la anticoncepción utilizó una literatura que argumentaba el origen hereditario del vicio, la indigencia, la enfermedad, la demencia, la idiocia, la criminalidad, de forma que necesitaba advertir, a pie de página, "No estamos de acuerdo en todo con los autores" (Lazarte, 1934a); pero no explicó las razones ni los elementos del desacuerdo. $\mathrm{Y}$, aunque fuese para prestigiar la anticoncepción y dándole un sentido diferente a la pretensión del predominio de los más favorecidos, arrastró la retórica de la llamada por Anne Carol "eugenesia aristocrática" (Ledesma, 2012, p. 158) cuando sostenía que "sólo los pobres e ignorantes", "campesinos pobres y gentes sin oficio" no usaban anticonceptivos", y apostillaba:

Por supuesto no es este contingente de niños de tan superior calidad - ni el Standard de vida admirable - como para aplaudir así no más, sin llamar la atención la prolificidad de estas clases si tenemos un verdadero amor por la raza hispanoparlante e interés por la grandeza biológica del porvenir [...] los grupos en mejores condiciones económicas y culturales son también los que más conocen los anticonceptivos y los que por otras razones procrean en general menos (Lazarte, 1934c).

Apoyándose en citas de Havelock Ellis (1859-1939) como "En la regulación de los nacimientos poseemos un estimable instrumento no sólo para la inmediata mejora de la sociedad sino para el enaltecimiento de la raza [especie] humana" (Lazarte, 1933e), utilizó una retórica eugenésica en su campaña en pro de los anticonceptivos que le llevó a legitimar el espaciamiento de los partos con dicha retórica, aconsejando "a las madres pobres no tener nuevos hijos antes de los cuatro años, y a las madres en buenas condiciones económicas, no hacerlo antes de los tres años. Medida que fortalecerá la esencia de una verdadera raza fuerte, base para un pueblo libre" (Lazarte, 1934b). Podemos apreciar que en la cita utilizada por Lazarte, Ellis proclamaba como medida la regulación de los nacimientos, cuestión central del neomaltusianismo, que supondría una mejora de la especie humana en su conjunto y no de un grupo social considerado superior ${ }^{22}$. En este mismo sentido utilizaba Lazarte el concepto de "raza", añadiendo dos vertientes del problema el biológico y el económico: "la raza se resiente en su biología, y sin una raza fuerte no conseguiremos crear una civilidad fuerte. El problema es biológico y económico y los dos factores es necesario extenderlos a las masas" (Lazarte, 1933e). Para Lazarte, la herencia no era más que un elemento de otros muchos: "son los trabajos rudos y malos para las madres, la miseria, la herencia e ignorancia los factores que determinan la alta mortalidad infantil y sobre los cuales podemos actuar" (Lazarte, 1933e).

De esta manera, se mezclaban en el discurso de Lazarte sobre la limitación de nacimientos neomaltusianismo con toques de eugenesia porque, en definitiva el objetivo último de las propuestas realizadas era no sólo disminuir la cantidad de nacimientos o aumentar la calidad biológica de los nacidos sino lograr una vida merecedora de ser vivida; de ahí la reproducción de la queja expresada por una mujer que con trece hijos nacidos y siete vivos decía: "No me ha quedado mucho tiempo para divertirme" ${ }^{\prime 23}$. No se trataba sólo de sobrevivir sino de tener una buena vida.

\section{LA REVOLUCIÓN SEXUAL DESEADA}

Como ya apuntamos en el epígrafe anterior, en la significación libertaria que Lazarte dio a la limitación de los nacimientos la liberación de las mujeres jugó un papel central. La contracepción permitiría a la mujer ser "fisiológicamente libre" (Lazarte, 1934c). Los anticonceptivos posibilitarían oponer el concepto de "emancipación biológica" frente al de "tragedia biológica" ya que llevarían a la libertad femenina para tener hijos "cuando quiera", y al derecho de la mujer a la limitación de los nacimientos sin "servidumbres ni subordinación" (Lazarte, 1933c). Oponiendo consciencia, inteligencia y ciencia a religión, se alumbraría una nueva mujer "la mujer de los 40" porque la mujer de los años 30 era una "mujer de transición" (Lazarte, 1933c). La técnica anticonceptiva significaba civilización, considerada ésta como difusión de conocimientos útiles y aspiración de hacer posible, para todos los miembros de la sociedad, una vida digna de ser humana; porque mientras la mujer fuese una "sierva de la ignorancia, miseria, marido e hijos no habrá redención humana posible" (Lazarte, 1933c). En este sentido, 
Lazarte era optimista al considerar que se había desarrollado una nueva ética sexual (neomaltusiana), la cual estaba muy vinculada e incluso supeditada al desarrollo de los avances científicos, porque, aunque el mismo Lazarte sostenía que "neomaltusianismo hubo en Egipto de Tut-Ank-Ammen, en China de Confucio, como en Roma de César" (Lazarte, 1934b), debido a la ineficacia de los medios que habían estado disponibles para procurar la anticoncepción, "el esfuerzo hondo de la nueva consciencia en separar proceso reproductor de ritual dé comunión física y espiritual: placer; en distinguir y disociar sexo de multiplicación [...] chocaba contra resultados que no deseaban, así el hombre como la mujer" (Lazarte, 1934b). Hasta entonces no se había llegado a una total liberación porque "los medios anticonceptivos eran inseguros" (Lazarte, 1934b). Pero en aquellos momentos la ciencia había logrado que se produjesen "resultados seguros en un cien por cien" y ya sólo faltaba "que la ciencia ponga en el comercio, al alcance de todos, en especial de las clases pobres, sus estupendas conquistas. ¡Ello no tardará mucho!" (Lazarte, 1934b). Y es aquí donde la ciencia se convertía en "un gran instrumento de liberación humana en lo tocante al amor" porque había posibilitado que "la mentalidad sexual de las masas", sobre todo de las mujeres jóvenes, cambiase ya que "el mayor descubrimiento de los siglos [era] la comprensión de que la mujer desea el amor sexual igualmente que el hombre" (Lazarte, 1933b). De esta forma se había conseguido "derrotar a las instituciones del orden actual", ya que

El matrimonio yacía desvencijado en descrédito creciente, reducido a los límites del prestigio burgués; la prostitución perdía terreno combatida enérgicamente, no por el Estado, su cómplice, ni por las clases privilegiadas que la necesitan, sí por la juventud femenina, el despertar erótico y la consciencia de los derechos de la mujer, desde un punto de vista extramatrimoniales" (Lazarte, 1934c).

La contracepción, como hemos dicho, permitiría a la mujer ser "fisiológicamente libre" y con ello nacería un mundo nuevo en el que la maternidad sería consciente y la prostitución y las enfermedades venéreas desaparecerían (Lazarte, 1934b).

Por otro lado, el poder desestabilizador del orden sexual dominante que poseía el discurso de Lazarte se centraba en el análisis crítico de las causas históricas de unas relaciones sociales que estructuraban una doble moral sexual. Ésta se reflejaba en las diferentes respuestas sociales desencadenadas por una misma conducta en función de si el sujeto agente era hombre o mujer ${ }^{24}$. Las causas por las que el castigo del adulterio estaba generizado respondían a razones religiosas, culturales y económicas. Lazarte señalaba que en occidente el judeo-cristianismo jugaba un papel relevante junto con las ideas de "raza pura" vinculada al judaís- mo. En cuanto a los argumentos económicos occidentales, apuntaba al entramado económico familiar que había hecho de la mujer una propiedad privada del hombre y establecido unas prácticas hereditarias que mantenían unas relaciones de género desigualitarias. De esta forma, Lazarte sostenía que el adulterio podía

dividirse en dos sectores: el adulterio del hombre y el de la mujer. En sus orígenes, el primero no tenía importancia; los hombres tuvieron libertades y facultades; señores y privilegiados dictaron la ley. El adulterio verdadero fue el de la mujer, como lo prueba la misma historia del Nuevo Testamento y como lo afirman las costumbres de la burguesía internacional reflejados en sus códigos y leyes. Recién en las legislaciones modernas, más humanas, entra a considerarse el adulterio del hombre como delito y a darle importancia, aunque en espíritu no tanto como al de la mujer. [...] Moralmente, siempre ha pesado más sobre la mujer que sobre el hombre, porque éste exigió seguridades en la paternidad. La exigencia para la mujer de una monogamia rigurosa fue originada no sólo por el fín de dejar la propiedad, sino también por la seguridad que desearon los padres en tener, querer $y$ criar a sus hijos.

Sin embargo, según Lazarte, lo artificioso de esta situación había hecho que toda norma represora del adulterio resultara inútil de manera que "en el siglo XX el adulterio es una costumbre humana generalizada y lo suficientemente vitalizada para romper las normas de una vieja moral sexual". Resaltaba así cambios históricos que se debían a avances de la consciencia femenina, nuevos conocimientos científicos y cambios sociales y culturales. En relación al avance de la consciencia femenina, Lazarte maximizaba las nuevas corrientes de pensamiento que optaban por una liberación sexual que permitiera a las mujeres disfrutar de una sexualidad libre. Esta opción había llevado al "espíritu femenino" a mirar "científicamente los problemas del amor" y a declarar "que habiendo voluntad y libertad y no creándose consecuencias perjudiciales para terceros, todo acto sexual es moral". Además, en opinión de Lazarte, "infinidad de hombres piensan lo mismo". A este cambio en la subjetividad de las mujeres se habían unido reformas legales como el divorcio, y juntos habían transformado "el aspecto cerrado de la cuestión", rompiendo "el vínculo que parecía eterno" y dando "al amor territorios antes inexplorados". En definitiva Lazarte sostenía: "Si hoy muere [el adulterio como conducta delictiva] es porque principalmente lo mata la mujer, la juventud femenina, esos millones de seres que miran con desconfianza el matrimonio y defienden encarnizadamente sus libertades. Ellas le han concluido por hundir en el silencio".

En cuanto a los nuevos conocimientos científicos, Lazarte recurría a la autoridad de antropólogos como Malinowski para deconstruir el imaginario burgués hegemónico en occidente: 
Mas está demostrado, como lo comprueba Bronislaw Malinowski, que no es ese [asegurar la paternidad biológica] un instinto básico; en Melanesia los salvajes no tienen un concepto claro de la paternidad. Pueden querer un hijo creyéndole suyo, como pueden estimarle mayormente sabiéndolo de su mujer. Una de las bases de los sentimientos paternales es la convivencia juntos. Un hombre que ve nacer a un niño y desarrollarse, instintivamente le quiere. Esto no sólo se percibe en las islas de Trobiand, sino que lo estamos viendo en quienes sacan bebés del hospicio para criarlos.

De esta manera negaba la relación del adulterio como delito con los instintos o la inteligencia, considerándolo como "una excrecencia de los sistemas patriarcales, forma nacida del egoísmo cimentado por ideas religiosas o por ambiciones terrenas". Es más, considerando a la ciencia y la tecnología como herramientas liberadoras en tanto que moralizaban la libertad sexual, sostenía:

[El adulterio como delito] pertenece a épocas en que amor es reproducción, en las cuales no se concibe ni el amor placer o recreación y camaradería. Se origina en los tiempos en que se ignoraba los millones de espermatozoides que produce el hombre en un instante o los millones de óvulos que da periódicamente una mujer. De haberse inventado el microscopio mil quinientos años antes y los anticonceptivos aplicados en

el siglo XII, ya no lo consideraríamos en serio ${ }^{25}$.

En esta clave de enfrentar lo nuevo con lo viejo, Lazarte concluía que cambios sociales y culturales muy relevantes habían sido posible debido a determinados procesos de decadencia; "la decadencia del poder paterno, la decadencia de la sujeción de la mujer, la decadencia de la costumbre y pretensión de casarse con vírgenes", habían "colaborado eficazmente en la decadencia del adulterio como lacra, mal, pecado o delito" (Lazarte, 1932). Luego la decadencia también podía ser un motor de transformación social liberadora, si se trataba de la decadencia de instrumentos represores.

Además, no existía en la obra de Lazarte una ideología del progreso clara, pues, implícitamente, consideraba que se habían producido retrocesos en una imaginaria línea del tiempo ${ }^{26}$. Por ejemplo, en el caso del abordaje de la sexualidad en la era precristiana y cristiana que expusimos en anteriores páginas, donde el periodo cristiano habría supuesto un retroceso en la consideración de la sexualidad humana. Es más, consideraba que uno de los desarrollos de la civilización hebreocristiana, el sistema capitalista, había provocado tal degeneración ${ }^{27}$ que estaba conduciendo a un alto porcentaje de mujeres frígidas, consideradas anormales porque "la sensualidad es una forma normal de sensibilidad específica" y "la mujer en su estado de frigidez o anestesia está en inferiores condiciones biológicas, sociales y anímicas" (Lazarte, 1935a).

\section{Frente a este estado de cosas}

las mujeres en las razas salvajes son perfectamente normales. Es rarísima la que carece de sensualidad. Entre el hombre salvaje y su compañera hay una reciprocidad perfecta en sus relaciones erotipsicofísicas.

Es de notar que siendo normal la vida sexual en las mujeres salvajes no existe, salvo rarísimas excepciones consecuencias degenerativas" (Lazarte, 1935a).

Así, por una parte, la decadencia de una civilización basada en la pérdida de vigencia de sus supuestos sociales y culturales que otros habían interpretado en clave noventayochocentista (de ocaso cultural), también podía ser entendida como un proceso de alumbramiento de una sociedad más igualitaria, y, por otra parte, siempre cabría la posibilidad de que dicho proceso supusiera una marcha hacia la opresión y la explotación.

No se trataba por tanto de un problema de regresión hacia estadios más primitivos de la especie humana sino todo lo contrario: una de las vías de la civilización, la capitalista (no la civilización en sí misma), era la que provocaba la degeneración de la especie humana, en este caso ejemplificada en la sexualidad anormal representada por la frigidez en las mujeres. La esencia del problema era la disarmonía, las "contradicciones profundas" del sistema (Lazarte, 1935a). Por eso, "al tener en cuenta la potencia erótica en la mujer primitiva y la degeneración de la mujer moderna, necesitamos buscar las causas que han establecido y están estableciendo esta degeneración y la encontramos menos en el orden psicológico que en la contextura social", de manera que

[la situación] se ha agravado enormemente por las condiciones a que el capitalismo la sometió sobre todo en su fase industrial y de racionalización. Masas respetables de mujeres tienen a la fuerza que aceptar trabajo sedentario, no hay otra manera de vivir: costureras, textiles, cigarreras, modistas, empaquetadoras, empleadas, bolseras, dactilógrafas, etc., en malas condiciones higiénicas y en malas condiciones económicas que, a su vez, son semejantes a las de la vida fuera del trabajo.

La influencia del ambiente, la economía y el trabajo (en pequeña escala la herencia) han creado una incapacidad sexual -atrofia de la sensualidad- en grandes masas de mujeres de las clases trabajadoras [y, por otra parte], en las clases adineradas, las mujeres han tomado también la dirección de una vida de holgazanería y ocio que si es verdad que a prima facie parecería lleva a un camino de hipersexualidad, en cambio arrastra infinidad de veces a la impotencia femenina por razones psicológicas y económicas, pues en estas clases hasta hace poco tiempo la única salida del sexo era el matrimonio (por lo menos la salida regular). Dentro de esta categoría y abarcando una clase media se encuentra como causa de la degeneración que venimos analizando el uso de los tóxicos, 
alcoholes, la frecuentación de cabarets, boites, donde entre la danza y el flirt, no se llega a ningún lado, fuera de la exasperación de los instintos, arsenal moderno que alimenta y vivifica al tipo de la 'flapper', que es un caso clavado de decadencia femenina... (Lazarte, $1935 a)^{28}$.

Así se llevaba a las mujeres a "ese extremo": "una frigidez de grandes masas de mujeres", al mismo tiempo que se iba hacia "una exacerbación del sexo, en un sentido de preparación y de aumento patológico que abarca a la generalidad de los hombres obligando a una mayor demanda femenina, práctica o teórica, por su parte", correlacionándose esto con la prostitución donde se unían "los instintos sexuales con el dinero" (Lazarte, 1935a).

La solución a esta situación no era individual sino colectiva, puesto que colectivo era el problema: actuar sobre "un mundo de desigualdad económica y prejuicios sexuales" por medio de la educación sexual. "Sólo así podremos retornar a una sensualidad natural, sana y altamente benéfica para la naturaleza humana" (Lazarte, 1935a).

\section{EL CONOCIMIENTO A TRAVÉS DEL DEBATE DE LAS IDEAS}

Ya hemos hecho mención del reconocido interés de Lazarte por la discusión de las ideas y la sintonía, en este sentido, con la política editorial de Estudios. Basta hacer un breve análisis comparativo de los artículos de Juan Lazarte "Desprestigio del adulterio" y el inmediatamente posterior en las páginas del número 105 de Estudios "La virilidad del hombre", de Julio Atarfe, para entender el propósito de la revista de establecer un proceso de reflexión y debate sobre las ideas existentes en torno a un tema. Mientras Lazarte consideraba que el adulterio era una costumbre humana que rompía con las normas de una "vieja moral sexual", criticaba la destrucción del amor recreación en la sociedad judeocristiana capitalista, y pregonaba la desaparición de la figura delictiva del adulterio, dado que la libertad sexual se imponía como un estado natural refrendado por la ciencia que había redescubierto el amor recreación ${ }^{29}$, Atarfe sostenía que la infidelidad, tenía casi siempre "por único acicate un deseo morboso que nada tiene que ver con el verdadero deseo amoroso; la infidelidad, causa siempre de disgustos y de tragedias matrimoniales, es frecuente en quienes probaron con exceso los placeres de fácil acceso y de moralidad dudosa antes de la unión formal" (Atarfe, 1932). Para evitarla, proponía, igual que Lazarte, dar a los jóvenes una buena educación sexual, pero esta vez el interés intrínseco ${ }^{30}$ era muy diferente, opuesto, pues con esta actuación se trataba de hacer comprender "que la función sexual tiene un fin más elevado que responder a la satisfacción de un apetito carnal; que dicho acto representa la perpetuación de la espe- cie [y que] el goce que proporciona no es más que el medio que la Naturaleza ha puesto a su alcance para la procreación de los hijos". Así, preconizaba la continencia sexual y consideraba una medida de eugenesia limitar la sexualidad a la reproducción (Atarfe, 1932). Como podemos apreciar, el debate estaba servido.

En cuanto a los contenidos de los textos de Lazarte, hemos de decir que de manera reiterada expresaban una marcada disposición a poner de manifiesto la existencia de distintas visiones de la realidad: "no todos los autores opinan lo mismo" de una determinada técnica anticonceptiva, o los efectos que presentaban eran "contradictorios" (Lazarte, 1934b). En el orden de los resultados subrayaba las divergencias que evidenciaban distintas realidades: "Nuestras encuestas no están de acuerdo con las de Europa" (Lazarte, 1935a), refiriéndose a la de países latinoamericanos.

Por otro lado, tras exponer sus consideraciones y argumentos sobre un tema, no tenía problemas en declarar que había "varias y encontradas opiniones" sobre dicho asunto (Lazarte, 1933a). De la misma manera, mostró las distintas posiciones acerca del apoyo de los profesionales de la medicina a los anticonceptivos que había conocido a través de un sondeo que él realizó:

De 20 médicos versados en estas cuestiones por su especialidad o vocación a quienes hice preguntas alusivas a estos casos, 15 contestáronme que sólo a requerimiento y en determinadas condiciones de la clientela, dan instrucciones; cinco que no las dan nunca. Mas el porcentaje aumenta todavía y el acuerdo reina casi en la mayoría de los profesionales jóvenes cuando las preguntas engloban temas teóricos y se averigua, por ejemplo, si son partidarios de creación de clínicas de enseñanza anticoncepcional (públicas y controladas por instituciones sanitarias nacionales o municipales), en su casi totalidad responden afirmativamente" (Lazarte, 1934c).

Ante esta situación, concluía que no quería con esto manifestar ninguna opinión colectiva ya que "cada profesional debe en esta hora tener ideas concretas sobre tales tópicos y allá cada uno con los suyos..." (Lazarte, 1934c).

No sólo opiniones y argumentos podían ser diferentes sino también los datos supuestamente objetivos, así las estadísticas, eran motivo de múltiples interpretaciones; aunque sostenía que los intervalos de dos o tres años entre un parto y otro permitirían bajar la mortalidad infantil como lo confirmaban las cifras que él presentaba, planteó que "estas estadísticas pueden prestarse a controversia" ya que "no puede ser el factor periodicidad el único determinante o valedero, pero sí es uno de los coadyuvantes" (Lazarte, 1934b).

En este intento de mostrar y debatir sobre las ideas, Lazarte no sólo aclara a los lectores que no estaba de 
acuerdo "en todo" con los autores que citaba (Lazarte, 1934a), sino que también dialogaba con su propia visión de las medidas y concepciones que desde supuestos eugenésicos se estaban poniendo en marcha. Utilizó una retórica eugenésica que incluía tintes represores, en defensa de los anticonceptivos, ya que concluía que "estará moralmente justificada la aplicación $^{31}$ o consejo de anticonceptivos en la serie de enfermedades susodichas" y el médico tendría en ello "la más grande responsabilidad" como "obrero social" que era (Lazarte, 1934a). Sin embargo, criticaba el uso político de la anticoncepción por esterilización:

La esterilización por medio de rayos $\mathrm{X}$ es un método usado principalmente en enfermos sin remedio y practicado en algunos Estados modernos con degenerados, imbéciles, criminales y tarados. Fue experimentado en Indiana, año 1907; Suiza, 1913; en Dinamarca, en 1927. Es un método un poco bárbaro, pues puede prestarse a errores fundamentales, sobre todo, si se admite el criterio político de los que han monopolizado la moral" (Lazarte, 1934b) ${ }^{32}$.

En esta dialógica que estableció Lazarte consigo mismo hacía participar, de manera indirecta, a los agentes implicados en una determinada cuestión. Así, afirmaba: "la inmoralidad de que las madres tengan un hijo cada año", y después puntualizaba: "este postulado que parece extraño y tal vez exagerado se deduce lógicamente de las nuevas conclusiones de la Ciencia" (estas conclusiones eran que una alta natalidad conllevaba una alta mortalidad infantil y maternal, y una baja natalidad conllevaba una baja mortalidad infantil y maternal) (Lazarte, 1934b). Ahora bien, la ciencia no era la única vía de conocimiento y Lazarte dio una gran relevancia a la experiencia de los no-expertos ${ }^{33}$ : las madres tenían "la intuición de estas verdades", y "cuanto el sentido común percibió desde lejos lo viene a comprobar la ciencia" (Lazarte, 1934b) ${ }^{34}$.

Su pensamiento dialógico le llevó no sólo al uso continuo de la intertextualidad ${ }^{35}$ sino también a pensar como compatibles planteamientos en aparente oposición, aunque sin obviar las diferencias: "aunque la ciencia esté subordinada al capitalismo, la Humanidad consigue, alcanza algo, en el sentido de su liberación; al fin, la burguesía no se llevará a su tumba histórica los descubrimientos de Pasteur, la televisión o el Zeppelín" (Lazarte, 1936). En este sentido abordaba el debate entre las doctrinas psicosomática y psicoanalítica. Por una parte, Mayer, Weber, Bernheim, Marchand exponiendo cómo los influjos psíquicos $y$ afectivos pueden actuar sobre el funcionamiento del cuerpo mediante sugestión; por otra parte, "la escuela psicoanalítica" sosteniendo la existencia del subconsciente; mientras él consideraba que ese debate en términos dicotómicos no era necesario ${ }^{36}$ : "No entraremos a verificar cuál de las dos doctrinas tiene razón entera; el caso es una explicación lógica, natural y confirmada por la experiencia y, por lo tanto, por el pensamiento científico"; y, además, añadía: "No es suficiente el criterio psicológico; existe otro que podríamos llamar anatomofisiológico" (Lazarte, 1936). Esta lógica también la aplicaba explícitamente cuando trataba de explicar las doctrinas maltusiana y neomaltusiana (Lazarte, 1934b). Lo relevante para Lazarte era que la limitación de los nacimientos había estado bloqueada porque las propuestas de Malthus estaban cargadas "de una cantidad de prejuicios y bajo el clima moral de su época sirvió a la reacción, y ésta se sirvió de sus ideas y doctrinas para la sujeción de las clases pobres" (Lazarte, 1934b). Lo que hizo Malthus, en palabras de Lazarte fue dar

un argumento científico cuando era más necesario para justificar la hegemonía eterna de las clases adineradas, muchos años después de la Revolución francesa [...] Sexualmente implicaba la subsistencia de un proletariado, la conformidad de estos esclavos con su estado y hasta un principio de libertad de reproducción basado en la riqueza ${ }^{37}$ [...] Este conjunto de arbitrariedades fue tomado como ciencia (Lazarte, 1934b).

Esta crítica de Malthus se apoyaba en las afirmaciones de algunos de sus opositores (Marx, William Godwin, Francis Plate, Hildegart) y le servía a Lazarte para introducir el neomaltusianismo, exponiendo las diferencias entre una y otra opción: el maltusianismo preconizaba la moral restrain o castidad, mientras el neomaltusianismo preconizaba los métodos anticonceptivos más científicos y modernos ${ }^{38}$. Pero también existían semejanzas: el reconocimiento de la necesidad de la limitación de la población, razón por la que "Malthus era neomaltusiano" (Lazarte, 1934b).

\section{CONCLUSIONES}

La consideración del neomaltusianismo como mero apéndice anticoncepcional de la eugenesia, diluye el papel que tuvo el movimiento anarquista en el desarrollo y alcance del mismo. Sin embargo, la puesta en evidencia de un uso táctico de la retórica eugenésica en la propuesta neomaltusiana contribuye a visibilizar el grado de complejidad que la dinámica social imprimió a la teoría y la praxis en torno a los conceptos de eugenesia y neomaltusianismo. El análisis de la obra de Juan Lazarte publicada en las páginas de Estudios nos ha permitido mostrar cómo se legitimó y reivindicó la significación libertaria de la limitación de los nacimientos en todos los ámbitos de la vida (económico, social, biológico y moral) y, sobre todo, en la liberación de las mujeres.

La eugenesia en el anarquismo no fue una contaminación burguesa sino un instrumento que acabó siendo resignificado. Sin duda, podemos discutir el éxito de dicha resignificación, pero sólo a la luz de los 
acontecimientos posteriores. En el discurso libertario de las primeras décadas del siglo XX, los elementos de la eugenesia fueron recursos para apoyar la defensa de una anticoncepción con la que se pretendía el desarrollo de una buena vida. El discurso de Lazarte puede enmarcarse en esas teorías del buen vivir que concebimos como propuestas incluyentes que como todo proceso de inclusión tiene su reverso de exclusión; en este caso excluyen las visiones mecánicas del mundo a través de una dialógica en la que caben contradicciones. Así, el neomaltusianismo no sería un instrumento de la eugenesia sino una táctica para "el buen vivir".

También podemos resaltar la participación de los textos analizados en la generación de un saber alternativo que incluiría la visión del "otro" mediante distintas estrategias de intertextualidad, desde citas directas e indirectas de expertos y legos a la recogida y elaboración de opiniones sobre los temas tratados. Por tanto, este análisis pone de manifiesto que no es posible abordar el pensamiento anarcolibertario en términos dicotómicos, como ya hemos expuesto en anteriores trabajos, pues buena parte de su producción discursiva se ha desarrollado sobre una dialógica. En el caso de Lazarte, en este proceso era fundamental el contexto histórico en el que se desenvolvían las cuestiones relativas a la vida de los seres humanos, de ahí que en sus escritos siempre aportara un recorrido histórico más o menos extenso del asunto que trataba. Al no concebir la historia desde una ideología del progreso y empeñarse en considerar los distintos aspectos de los problemas (biológicos, económicos y culturales), el discurso de Lazarte en Estudios contribuía a conformar un mundo que desafiaba los supuestos deterministas.

\section{AGRADECIMIENTOS}

Esta investigación forma parte de los proyectos HAR2009-13389-C03-01 y HAR200913389-C03-03 financiados por el Ministerio de Economía y Competitividad.

\section{NOTAS}

1 Nos interesa especialmente el ámbito argentino y el ámbito español donde la importancia de los proyectos eugenésico y neomaltusiano ha sido ampliamente tratada por Marisa Miranda y Gustavo Vallejo para el caso argentino y por Raquel Álvarez y Eduard Masjuan i Bracons para el caso español. Puede verse una reciente y breve síntesis de los abordajes que se han realizado sobre la eugenesia en Argentina en Ledesma, 2012, pp. $153-164$

2 Esta diversidad se ha trasladado a la historiografía de forma que se ha interpretado de diferentes maneras las relaciones entre el neomaltusianismo y la eugenesia en el ámbito anarquista español. En Girón, 2005, pp. 23-28, se hace una magnífica síntesis y se ofrece una amplia bibliografía de este panorama en el que, entre la afirmación de la existencia de una clara relación entre neomaltusianismo y eugenesia en el movimiento libertario en España y la consideración de que no estarían vinculadas, caben distintas posiciones y matices.

3 Para el conocimiento de este procedimiento nos ha sido muy útil el trabajo de Martín, 1996-1997, pp. 1-37.

4 En el libro colectivo Lida y Yankelevich, 2012, pueden verse diversos estudios acerca de las resistencias y las alternativas libertarias a la cultura burguesa dominante; para el caso argentino donde Lazarte desarrollaba su actividad profesional y política, véase el capítulo de Suriano, 2012.

5 Ha sido profusamente señalada la diversidad de corrientes en el seno del movimiento anarquista, por lo que aquí sólo seña-

laremos que con esta denominación no pretendemos hacer referencia a un discurso anarquista único, como, por otra parte, quedará explícito en la consideración de la práctica discursiva anarquista como esencialmente dialógica, o en la pluralidad de posiciones ante temas como el neomaltusianismo a las que hacemos mención más adelante.

6 Algunos de los estudios que han abordado el análisis de los objetivos de las ediciones ácratas son: Graciano, 2012; Navarro, 1997. Sobre la autogestión del conocimiento a través de prácticas comunicativas en la prensa libertaria puede verse Tabernero, Jiménez y Molero, 2013.

7 Para tener una perspectiva general de la revista puede verse el magnífico trabajo de Navarro, 1997.

8 Sobre difusión de Estudios en Argentina, ver Navarro, 1997, pp. 70-71.

9 Rosario, ciudad natal de Juan Lazarte, es considerada la segunda ciudad argentina en relación a la importancia que adquirió la influencia libertaria. Juan Suriano califica de mítico el círculo libertario «Ciencia y Progreso» que desarrolló una «prolífica actividad» en esa ciudad durante los años finales del siglo XIX e iniciales del XX (Suriano, 2004).

10 Julio Barcos (Santa Fe, 1883 - Buenos Aires, 1960) fue un maestro vinculado al movimiento libertario argentino, que tuvo fuertes lazos con integrantes españoles del proyecto pedagógico de la Escuela Moderna (Suriano, 2004, p.242), y que fue colaborador de la revista Estudios con una sección titulada «Gacetilla». 
Sobre la influencia de Barcos en Juan Lazarte puede verse Ledesma, 2010a. Acerca de las conexiones entre el anarquismo español y argentino puede verse Moya, 2010.

11 Más detalles sobre la biografía de Juan Lazarte pueden encontrarse en: Abad, Invaldi y Cappelletti, 1964; y en Ledesma, 2010b.

12 Julio Barcos escribió el libro La libertad sexual de las mujeres que ha sido considerado un precedente en el tratamiento de este tipo de temas por parte de autores como Lazarte; véase Ledesma, 2010a, pp. 2, 4-5.

13 Suriano describe cómo la exposición de controversias y su debate en torno a diversos temas se desarrollaron a finales del siglo XIX y principios del XX en los círculos anarquista argentinos (Suriano, 2004, pp. 125-128; Suriano, 2005, p. 45).

14 Este fragmento ha sido citado anteriormente en Ledesma, 2010b.

15 En este sentido, la denuncia en la prensa escrita, como expresión máxima del descontento social, era un instrumento fundamental para lograr el desmantelamiento del sistema social capitalista y su transformación revolucionaria, al poner en evidencia las causas de los problemas que padecían las clases más desfavorecidas. Véase Jiménez, 1995, pp. 35-44. Como se sostenía en Jiménez, 1998, p. 300, la prensa anarquista estaba obligada a poner de manifiesto las infamias capitalistas y cada artículo relacionado con la sanidad debía ser «una acusación mortal para el régimen burgués». Fantasma [Javier Serrano]. Ciencia y revolución. Solidaridad Obrera, 17 julio 1932. También puede verse el protagonismo que tuvieron las denuncias en Molero y Jiménez, 2013, pp. 34-38.

16 Dora Barrancos (pionera en los estudios sobre anarquismo y sexualidad en Argentina) ha señalado en diversas ocasiones la importancia que los integrantes del movimiento anarquista argentino tuvieron en las propuestas de cambio de la moral y la conducta sexual (Barrancos, 1990, 2001 y 2006).

17 Sobre el debate interno que se produjo en el anarquismo español en pro y en contra del neomaltusianismo, véase Masjuan, 2000, pp. 34-44.

18 En 1934 la editorial Argos de Barcelona (España) publicó Limitación de los Nacimientos: contribución al estudio de los problemas sexuales que en una edición posterior de la Librería Ruiz de Rosario (Argentina), en 1936, pasaría a denominarse Contralor de los nacimientos.

19 El pedagogo anarquista francés Paul Robin, considerado uno de los más importantes elaboradores y defensores del neomaltusianismo, abanderó la reivindicación del placer sexual como elemento esencial para una vida sana así como el derecho al conocimiento y la autogestión del propio cuerpo (MacLaren, 2000, 175-176). Estas ideas generaron una corriente dentro del movimiento libertario (Accampo, 2003) en la que Lazarte puede ser incluido a tenor de sus publicaciones en Estudios.

20 En un artículo publicado en el no 141 sostenía «todo el mundo sabe que salvo en un pequeño número de casos, en que diferentes taras somáticas se suman, la fecundación en el femenino humano no depende de la sensualidad». Por ejemplo, como sostenía Lazarte en dicho artículo, la frigidez femenina podía coexistir con la maternidad (Lazarte, 1935a).
21 Como ha sido puesto de manifiesto en múltiples ocasiones, eugenesia es un término polisémico; sobre sus diversos significados puede verse Paul, 1995. La eugenesia galtoniana está muy ligada a la herencia de rasgos físicos y conductuales, no así otras variantes de la eugenesia. Sobre la eugenesia en el anarquismo pueden verse Cleminson, 2008; Molero y Jiménez, 2010; Ledesma, 2010a y 2012.

22 La deriva jerarquizante de la propuesta de Galton vendría avalada por la consideración de «more suitable races» y «less suitable races» (Galtón, 1907, p. 17). Sin embargo, la resignificación de la eugenesia en un sentido liberador propugnada por el anarcolibertarismo consideraba la noción de raza como sinónimo de especie (Vallejo y Miranda, 2004, p. 429). El carácter inclusivo de la llamada eugenesia progresista les permitió, como ha señalado Marisa Miranda, identificar raza con especie humana (Miranda, 2005, p. 193). Sin embargo, la práctica hegemónica de la eugenesia no permitió dicho desarrollo, utilizándola como una herramienta de reforzamiento de la jerarquización social.

23 Ya hemos tratado en páginas anteriores este caso que fue incluido en Lazarte, 1934a.

24 El primer artículo de Lazarte publicado en Estudios ponía en evidencia esta realidad respecto a la conducta de adulterio (Lazarte, 1932). Todas las citas que siguen hasta la siguiente nota corresponden a este artículo.

25 Lazarte, 1932. En nuestros anteriores análisis de Estudios pudimos apreciar que las colaboraciones del médico argentino Juan Lazarte en la revista jugaron un interesante papel en la consideración de la ciencia y la tecnología como herramientas para la liberación sexual. Véanse Jiménez y Molero, 2009; y Jiménez y Molero, 2011.

26 Se han escrito muchas páginas sobre la asimilación de la ideología del progreso en el movimiento anarcolibertario, pero también, aunque no tan extensamente, se ha puesto en evidencia el cuestionamiento y la desconfianza en una historia lineal progresiva vinculada a una ciencia mitificada por el positivismo (Girón, 2005, pp. 64-65; Jiménez y Molero, 2009; Molero y Jiménez, 2010; Jiménez y Molero, 2011; Ardillo, 2013).

27 Aquí cabe señalar que mientras la «degeneración» que describía una regresión o retorno a una situación similar a la del ser humano primitivo tenía entre sus ejemplos más utilizados a las clases populares, en el anarquismo la armonía primitiva no era un estado inferior, por tanto el regreso a estadios más naturales no era considerado necesariamente negativo. En general, la lectura que hacían los diferentes socialismos del degeneracionismo imputaba el proceso a las condiciones generadas por el capitalismo y, por tanto, responsabilizaba del mismo a la burguesía (Girón, 2005, pp. 285-369 se hace una revisión de la evolución del concepto de degeneración, recogiendo una amplia bibliografía elaborada por Rafael Huertas y Ricardo Campos sobre el tema, en la que destaca Campos, Huertas y Martínez, 2000). El anarquismo resignificó de manera inmediata el término utilizándolo en la explicación de la lucha de clases. Lazarte, considerando la degeneración como un deterioro físico y psicológico, consecuencia del modelo social y cultural capitalista, utilizó el concepto en la explicación de la cuestión social (lucha de clases) y de la cuestión sexual (lucha por la emancipación de las mujeres).

28 Las últimas frases de esta cita evidencian la relación compleja que se mantenía en ámbitos anarquistas como Estudios con la 
denominada «mujer moderna». Las críticas más incisivas a este modelo de mujer hacían hincapié en sus hábitos burgueses y en las limitaciones de ese modelo para la verdadera emancipación de las mujeres. Sobre este asunto, véase Jiménez, 2011.

29 Véanse los epígrafe "La revolución sexual deseada» y «Significación libertaria de la limitación de nacimientos» en este artículo.

30 Eric Olin Wright en su teoría sobre las relaciones entre los grupos sociales (clases) ha definido dos tipos de intereses: intrínsecos e intrumentales. El primero se refiere a los fines de la acción y el segundo a los medios para alcanzar esos fines. Véase Jiménez, 1995, p. 12.

31 Las cursivas son nuestras, la llamada de atención se debe a que es este término el que da una connotación de no voluntariedad.

32 Nadia Ledesma ha puesto de manifiesto que en los años 40 Lazarte apoyaba, sin ningún tipo de cuestionamientos, las esterilizaciones (Ledesma, 2012, p. 161). Sería necesario ahondar en el análisis de las razones por las que se produjo este supuesto cambio de opinión, que Marisa Miranda (2011, p. 132) considera una inexplicable adscripción a la eugenesia de Bernardo de Quirós.

33 En general, Lazarte daba una gran relevancia a la experiencia y utilizaba la experiencia propia (en plural mayestático)

\section{BIBLIOGRAFÍA}

Abad de Santillán, Diego; Invaldi, Ángel; Cappelletti, Ángel J. (1964), Juan Lazarte, militante social, médico, humanista, Rosario, Grupo Editor de Estudios Sociales.

Accampo, Ellinor A. (2003), "The Gendered Nature of Contraception in France: Neo-Malthusianism, 1900-1920", Journal of Interdisciplinary History, 34(2), 2003, pp. 235-262.

Anónimo (1934), "Bibliografía. Cuadernos Economicos. Ediciones Imán, Buenos Aires”, Estudios, no 136, diciembre, p. 31.

Ardillo, José (2013), “La discusión sobre ecología, progreso y anarquismo a la luz del siglo XXI", Enciclopèdic, no 38, pp. 8-13.

Atarfe Castillejos, Julio (1932), "La virilidad del hombre", Estudios, no 105, mayo, pp. 21-23.

Barrancos, Dora (1990), "Anarquismo y sexualidad". En: Diego Armus (compilador), Mundo urbano y cultura popular. Estudios de historia social argentina, Buenos Aires, Editorial Sudamericana, pp. 15-37.

Barrancos, Dora (2001), "Moral sexual, sexualidad y mujeres trabajadoras en el período de entreguerras". En: Devoto, Fernando y Madero, Marta (directores), Historia de la vida privada en la Argentina, vol. 3, Buenos Aires, Taurus, 2001.

Barrancos, Dora (2006), "Problematic Modernity: Gender, Sexuality, and Reproduction in Twentieth-Century Argentina", Journal of Women's History, 18 (2), pp. 123-150. como fuente de autoridad. Véanse en este sentido Lazarte, $1935 b$ y 1936.

34 La experiencia lega la utilizó Lazarte en diversas ocasiones con expresiones como «muchas mujeres» o "una madre muy digna de confianza» (Lazarte, 1936, pp. 17-19).

35 Lazarte recurrió continuamente a autoridades en diversos campos del conocimiento, desde lo religioso (Evangelio de San Juan, la Biblia) a las ciencias biológicas pasando por la antropología y la psicología, para discutir y apoyar ideas y prácticas concretas.

36 Sobre la superación de la lógica dicotómica en el pensamiento libertario, véase Jiménez y Molero, 2009; y Jiménez y Molero, 2011

37 Esta crítica supone una ruptura con el principio de eugenesia aristocrática que señalábamos en páginas anteriores.

38 Lazarte mencionaba en este punto a Hildegart cuando sostenía que "Malthus no era maltusiano» en lo que se refería a los medios para lograr solucionar el problema que describía. Esta aserción la recogía Hildegart de Víctor Robinson quien se refería a la falta de efectividad de las propuestas de Malthus para acabar con el excesivo crecimiento de la población. Véase Lazarte, 1934b y Hildegart, 1931, p. 23.

Campos Marín, Ricardo, Martínez Pérez, José y Huertas García-Alejo, Rafael (2000), Los ilegales de la naturaleza. Medicina y degeneracionismo en la España de la Restauración (1876-1923). Madrid, CSIC.

Cappelletti, Angel J. (1964), "Juan Lazarte, un humanista". En: Abad de Santillán, Diego; Invaldi, Angel; Cappelletti, Angel J., Juan Lazarte, militante social, médico, humanista, Rosario, Grupo Editor de Estudios Sociales, pp. 33-41.

Cleminson, Richard (2008), Anarquismo y sexualidad en España, 1900-1939. Cádiz: Servicio Publicaciones Universidad de Cádiz.

Diez, Xavier (2007), El anarquismo individualista en España (19231938). Barcelona, Virus editorial, p. 132-4.

Galtón, Francis (1907 [1883]), Inquiries into Human Faculty and its Development (2a ed.). London, Dent \& Dutton, [en línea], disponible en: http://www.mugu.com/galton/books/human-faculty/.

García Birlán, Antonio (1936), “Bibliografía. La reforma universitaria. Líneas y trayectoria, por el doctor Juan Lazarte”, Estudios, no 150, marzo, pp. 30-31.

Girón Sierra, Álvaro (2005), En la mesa con Darwin. Evolución y revolución en el movimiento libertario en España (1869-1914). Madrid, CSIC.

Graciano, Osvaldo (2012), “La escritura de la realidad. Un análisis de la tarea editorial y del trabajo intelectual del Anarquismo argen- 
tino entre los años 30 y el Peronismo", [en línea], disponible en: http: //www.izquierdas.cl, [consultado el 12/4/2012], pp. 72-110.

Hildegart (1931), La revolución sexual. Valencia, Cuadernos de Cultura.

H.N.R [Higinio Noja Ruiz] (1932), "La revolución sexual de nuestro tiempo por Juan Lazarte”, Estudios, no 109, septiembre, p. 39.

Jiménez Lucena, Isabel (1995), Cambio político y alternativas sanitarias: El debate sanitario durante la Segunda República [tesis doctoral]. Málaga: Universidad de Málaga.

Jiménez Lucena, Isabel (1998), “La cuestión del 'regeneracionismo' sanitario y su debate durante la Segunda República: elementos de clase e ideología", Dynamis, 18, pp. 285-314.

Jiménez Lucena, Isabel y Molero Mesa, Jorge (2009), “Problematizando el proceso de (des)medicalización. Mecanismos de sometimiento/autogestión del cuerpo en los medios libertarios españoles del primer tercio del siglo XX". En: Miranda, Marisa y Álvaro Girón Sierra (coords.), Cuerpo, biopolítica y control social: América Latina y Europa en los siglos XIX y XX, Buenos Aires, Siglo XXI Ed Iberoamericana, pp. 69-93.

Jiménez-Lucena, Isabel (2011), “La inclusión excluyente y la razón que la interpela. La naturaleza de "la mujer nueva" en la prensa libertaria española de entreguerras". En: Porras Gallo, María Isabel, Berta Gutiérrez Rodilla, Mariano Ayarzagüena Sanz y Jaime de las Heras Salord, (eds), Transmisión del conocimiento médico e internacionalización de las prácticas sanitarias: una reflexión histórica, Ciudad Real, SEHM y Universidad de Castilla-La Mancha, pp. 395-399.

Jiménez Lucena, Isabel y Molero Mesa, Jorge (2011), "Good birth and good living. The (de)medicalising key to sexual reform in the anarchist media of inter-war Spain", International Journal of Iberian Studies (IJIS), 24 (3), pp. 219-241.

Lacerda de Moura, Maria (1932), "Procreación y miseria”, Estudios, no 106, junio, pp. 7-9.

Lazarte, Juan (1932), “Desprestigio del adulterio", Estudios, no 105, mayo, pp. 19-20.

Lazarte, Juan (1933a), “Significación cultural y ética de la limitación de los nacimientos", Estudios, no 120, agosto, pp. 14-16.

Lazarte, Juan (1933b), "Significación cultural y ética de la limitación de los nacimientos", Estudios, no 121, septiembre, pp. 28-29.

Lazarte, Juan (1933c), "Significación cultural y ética de la limitación de los nacimientos", Estudios, no 122, octubre, pp. 12-14.

Lazarte, Juan (1933d), "Significación cultural y ética de la limitación de los nacimientos", Estudios, no 123, diciembre, pp. 28-30.

Lazarte, Juan (1933e), "Significación cultural y ética de la limitación de los nacimientos", Estudios, no 124, diciembre, pp. 29-31.

Lazarte, Juan (1934a), "Significación cultural y ética de la limitación de los nacimientos", Estudios, no 125, enero, pp.3-5.

Lazarte, Juan (1934b), "Significación cultural y ética de la limitación de los nacimientos", Estudios, no 126, febrero, pp.17-20.
Lazarte, Juan (1934c), "Significación cultural y ética de la limitación de los nacimientos", Estudios, nำ128, abril, pp. 28-30.

Lazarte, Juan (1935a), "La atrofia de la sensibilidad en las mujeres", Estudios, no 141, mayo, Barcelona, pp. 18-20.

Lazarte Juan (1935b), "Los tiempos agenésicos", Estudios, no 147, noviembre, pp. 13-14.

Lazarte, Juan (1936), “Milagros y Medicina”. Estudios, no 156, septiembre, pp. 17-19.

Ledesma Prietto, Nadia F. (2010a), “Eugenesia, anarquismo y emancipación femenina. Una aproximación a los discursos libertarios. Argentina (1930-1940)", Ponencia presentada en el Seminario Internacional Fazendo Género 9, agosto 2010, [en línea], disponible en: http://www.fazendogenero.ufsc.br/9/resources/anais/1278270454 ARQUIVO FAZENDO\%20NADIA\%20 FINAL\%5B1\%5D.pdf, [consultado el 3/6/2012].

Ledesma Prietto, Nadia (2010b), “Revisión de la categoría de intelectual a través de la figura de Juan Lazarte y sus intervenciones en el anarquismo de los 30", [en línea], disponible en: http:// es.scribd.com/doc/73321596/intelectual-lazarte-, [consultado el $7 / 5 / 2012]$.

Ledesma Prietto, Nadia (2012), "Eugenesia bajo la lupa de las investigaciones sociales argentinas", Intersticios. Revista Sociológica de Pensamiento Crítico, 6 (2), pp.153-164.

Lida, Clara E. y Yankelevich, Pablo (comp.) (2012), Cultura y política del anarquismo en España e Iberoamérica, México D.F., El Colegio de México.

Martí Ibáñez, Félix (1937), "Sanidad, Asistencia social y Eugenesia en la Revolución social española", Estudios, no 160, enero, p. 34.

Martín Rojo, Luisa (1996-1997), "El orden social de los discursos", Discurso, no 21-22, pp. 1-37.

Masjuan i Bracons, Eduard (2000), La Ecología humana en el anarquismo ibérico: urbanismo "orgánico" o ecológico, neomalthusianismo y naturismo social. Barcelona, Icaria.

McLaren, Angus (2000), "Reproduction and Revolution: Paul Robin and Neomalthusianism in France". En: Doland, Brian (ed.), Malthus, Medicine \& Morality: "Malthusianism" after 1978, Amsterdam/Atlanta, Rodopi, 165-188.

Miranda, Marisa (2005), "La biotipología en el pronatalismo argentino (1930 1983)", Asclepio, 57 (1), pp. 189-218.

Miranda, Marisa (2011), Controlar lo incontrolable. Una historia de la sexualidad en Argentina. Buenos Aires, Biblos.

Navarro Navarro, F.J. (1997), El Paraíso de la Razón. La revista Estudios (1928-1937) y el mundo cultural anarquista. València, Edicions Alfons el Magnànim.

Molero Mesa, Jorge; Jiménez Lucena, Isabel (2010), “«Otra manera de ver las cosas». Microbios, eugenesia y ambientalismo radical en el anarquismo español del siglo XX". En: Miranda, Marisa y Gustavo Vallejo (dirs.), Darwinismo social y eugenesia. Derivas de Darwin: cultura y política en clave biológica, Buenos Aires, Siglo XXI Ed Iberoamericana, pp. 143-164. 
Molero-Mesa, Jorge; Jiménez-Lucena, Isabel (2013), “"Brazo y cerebro»: Las dinámicas de inclusión-exclusión en torno a la profesión médica y el anarcosindicalismo español en el primer tercio del siglo XX", Dynamis, 33 (1), pp. 19-41.

Moya, José C. (2010), “El anarquismo argentino y el liderazgo español”. En: García Sebastián, Marcela (dir.), Patriotas entre naciones. Elites emigrantes en Argentina, Madrid, Editorial Complutense, pp. 361-373.

Paul, Diane B. (1995), Controlling Human Heredity: 1865 to the Present. New Jersey, Humanities Press.

Suriano, Juan (2004), Anarquistas: Cultura y política libertaria en Buenos Aires, 1890-1910. Buenos Aires, Ediciones Manantial.

Suriano, Juan (2005), Auge y caída del anarquismo: Argentina, 1880-1930. Buenos Aires, Capital Intelectual.
Suriano, Juan (2012), "Las prácticas culturales del anarquismo argentino". En: Lida, Clara E. y Pablo Yankelevich (comp.), Cultura y política del anarquismo en España e Iberoamérica. El Colegio de México, pp. 145-174.

Tabernero-Holgado, Carlos; Jiménez-Lucena, Isabel; Molero-Mesa, Jorge (2013), "Movimiento libertario y autogestión del conocimiento en la España del primer tercio del siglo XX: la sección "Preguntas y respuestas» (1930-1937) de la revista Estudios", Dynamis, 33 (1), pp. 43-67.

Vallejo, G. y Marisa Miranda, (2004), "Los saberes del poder: eugenesia y biotipología en la Argentina del siglo XX", Revista de Indias, 64 (231), pp. 425-444. 